\title{
Dynamically generated inflation from non-Riemannian volume forms
}

\author{
D. Benisty ${ }^{1,2, a}$, E. I. Guendelman ${ }^{1,2,3, b}$, E. Nissimov ${ }^{4, c}$, S. Pacheva ${ }^{4, d}$ \\ ${ }^{1}$ Physics Department, Ben-Gurion University of the Negev, 84105 Beer-Sheva, Israel \\ ${ }^{2}$ Frankfurt Institute for Advanced Studies (FIAS), Ruth-Moufang-Strasse 1, 60438 Frankfurt am Main, Germany \\ ${ }^{3}$ Bahamas Advanced Study Institute and Conferences, 4A Ocean Heights, Hill View Circle, Stella Maris, Long Island, Bahamas \\ ${ }^{4}$ Institute for Nuclear Research and Nuclear Energy, Bulgarian Academy of Sciences, Sofia, Bulgaria
}

Received: 16 June 2019 / Accepted: 15 September 2019 / Published online: 28 September 2019

(C) The Author(s) 2019

\begin{abstract}
We propose a simple modified gravity model without any initial matter fields in terms of several alternative non-Riemannian spacetime volume elements within the metric (second order) formalism. We show how the nonRiemannian volume-elements, when passing to the physical Einstein frame, create a canonical scalar field and produce dynamically a non-trivial inflationary-type potential for the latter with a large flat region and a stable low-lying minimum. We study the evolution of the cosmological solutions from the point of view of theory of dynamical systems. The theory predicts the spectral index $n_{s} \approx 0.96$ and the tensor-to-scalar ratio $r \approx 0.002$ for $60 e$-folds, which is in accordance with the observational data. In the future Euclid and SPHEREx missions or the BICEP3 experiment are expected to provide experimental evidence to test those predictions.
\end{abstract}

\section{Introduction}

Developments in cosmology have been influenced to a great extent by the idea of inflation [1-5], which provides an attractive solution of the fundamental puzzles for the standard Big Bang model, as the horizon and the flatness problems. In addition, providing a framework for sensible calculations of primordial density perturbations were discussed in [6,7]. However, it has been recognized that a successful implementation requires some very special restrictions on the dynamics that drives inflation. In particular, in New Inflation [4], a potential with a large flat region, which then drops to zero (or almost zero) in order to reproduce the vacuum with almost zero (in

\footnotetext{
a e-mail: benidav@ post.bgu.ac.il

be-mail: guendel@bgu.ac.il

ce-mail: nissimov@inrne.bas.bg

de-mail: svetlana@inrne.bas.bg
}

Planck units) cosmological constant of the present universe, is required.

In a parallel development, extended (modified) gravity theories as alternatives/generalizations of the standard Einstein General Relativity are being extensively studied in the last decade or so. The main motivation for this development comes from:

- (a) Cosmology - modified gravity may solve the problems of dark energy and dark matter and explain the large scale structure and the accelerated expansion of the universe $[8,9])$;

- (b) Quantum field theory in curved spacetime - because of non-renormalizability of standard general relativity in higher loops it fails to describe the universe at quantum scales [10];

- (c) Modern String theory - because of the natural appearance of scalar-tensor couplings and higher-order curvature invariants in low-energy effective field theories aimed at phenomenologically realistic description of particle physics [11].

The principal approaches to construct modified gravity theories include $f(R)$-gravity, scalar-tensor theories, GaussBonnet gravity models. For detailed accounts, see the book [12] and the extensive reviews [13-16], as well as for further details Refs. [17-46].

One broad class of actively developed modified/extended gravitational theories is based on employing alternative nonRiemannian spacetime volume-forms, i.e., metric- independent generally covariant volume elements in the pertinent Lagrangian actions on spacetime manifolds with an ordinary Riemannian geometry, instead of the canonical Riemannian volume element $\sqrt{-g} d^{4} x$ whose density is given by the square-root of the determinant of the Riemannian metric: 
$\sqrt{-g} \equiv \sqrt{-\operatorname{det}\left\|g_{\mu \nu}\right\|}$

originally proposed in [47-51]. For a concise geometric formulation, see $[52,53]$.

This formalism was used as a basis for constructing a series of extended gravity-matter models describing unified dark energy and dark matter scenario [54,55], quintessential cosmological models with gravity-assisted and inflatonassisted dynamical suppression (in the "early" universe) or generation (in the post-inflationary universe) of electroweak spontaneous symmetry breaking and charge confinement [56-58], and a novel mechanism for the supersymmetric Brout-Englert-Higgs effect in supergravity [52].

In the present paper we propose a very simple gravity model without any initial matter fields involving several nonRiemannian volume-forms instead of the standard Riemannian volume element $\sqrt{-g} d^{4} x$. We show how the nonRiemannian volume-elements, when passing to the physical Einstein frame, generate a canonical scalar field $u$ and manage to create dynamically a non-trivial inflationary-type potential for $u$ with a large flat region for large positive $u$ and a stable low-lying minimum, i.e., $u$ will play the role of a dynamically created "inflaton". This dynamically generated inflationary potential turns out to be a generalization of the well-known Starobinsky potential [1].

We study the evolution of the cosmological solutions from the point of view of the theory of dynamical systems and calculate the spectral index $n_{s}$ and the tensor-to-scalar ratio $r$ in our model whose values are in accordance with the observational data.

In Sect. 2 below we briefly review the general notion of volume forms on arbitrary differential manifolds. Section 3 briefly presents the general construction of Lagrangian actions on Riemannian manifolds employing metricindependent (non-Riemannian) volume forms (volume elements). Our main results are contained in Sects. 4, 5 and 6. In Sect. 4 we propose our simple modified gravity model in terms of several non-Riemannian volume elements without any matter fields and derive the corresponding Einsteinframe description with the associated dynamical creation of a canonical scalar field with a non-trivial effective inflationary potential. In Sect. 5 we study the cosmological evolutionary solutions within the Friedmann-Lemaitre-Robertson-Walker framework. In Sect. 6 we derive the explicit expressions for the Hubble slow-roll parameters and use them to obtain analytic results for the scalar power spectral index and the tensorto-scalar ratio which we compare with the available observational data.The last Sect. 7 contains our conclusions.

\section{Non-Riemannian volume-forms formalism}

Let us first recall the general notion of volume-forms (volume elements) in integrals over arbitrary differentiable manifolds - not necessarily Riemannian one, so no metric is needed. Volume forms are given by nonsingular maximal rank differential forms $\omega$ (see e.g. Ref. [59]):

$$
\begin{aligned}
\int_{\mathcal{M}} \omega(\ldots) & =\int_{\mathcal{M}} d x^{D} \Omega(\ldots), \\
\omega & =\frac{1}{D !} \omega_{\mu_{1} \ldots \mu_{D}} d x^{\mu_{1}} \wedge \cdots \wedge d x^{\mu_{D}}, \\
\omega_{\mu_{1} \ldots \mu_{D}} & =-\varepsilon_{\mu_{1} \ldots \mu_{D}} \Omega,
\end{aligned}
$$

(our conventions for the alternating symbols $\varepsilon^{\mu_{1}, \ldots, \mu_{D}}$ and $\varepsilon_{\mu_{1}, \ldots, \mu_{D}}$ are: $\varepsilon^{01 \ldots D-1}=1$ and $\varepsilon_{01 \ldots D-1}=-1$ ).

The volume element density $\Omega$, as it is evident from its definition in (2), transforms as scalar density under general coordinate reparametrizations on the manifold.

In Riemannian $D$-dimensional spacetime manifolds a standard generally-covariant volume-form is defined through the "D-bein" (frame-bundle) canonical one-forms $e^{A}=$ $e_{\mu}^{A} d x^{\mu}(A=0, \ldots, D-1)$ :

$$
\begin{array}{r}
\omega=e^{0} \wedge \cdots \wedge e^{D-1}=\operatorname{det}\left\|e_{\mu}^{A}\right\| d x^{\mu_{1}} \wedge \cdots \wedge d x^{\mu_{D}} \\
\longrightarrow \Omega=\operatorname{det}\left\|e_{\mu}^{A}\right\|=\sqrt{-\operatorname{det}\left\|g_{\mu \nu}\right\|} .
\end{array}
$$

To construct modified gravitational theories as alternatives to ordinary standard theories in Einstein's general relativity, instead of $\sqrt{-g}$ we can employ one or more alternative nonRiemannian volume element densities as in (2) given by nonsingular exact $D$-forms $\omega=d A$ where:

$$
\begin{aligned}
& A=\frac{1}{(D-1) !} A_{\mu_{1} \ldots \mu_{D-1}} d x^{\mu_{1}} \wedge \cdots \wedge d x^{\mu_{-1}} \\
& \longrightarrow \quad \Omega \equiv \Phi(A)=\frac{1}{(D-1) !} \varepsilon^{\mu_{1} \ldots \mu_{D}} \partial_{\mu_{1}} A_{\mu_{2} \ldots \mu_{D}} .
\end{aligned}
$$

Thus, the non-Riemannian volume element density $\Phi(A)$ is defined in terms of the (scalar density of the) dual fieldstrength of an auxiliary rank $D-1$ tensor gauge field $A_{\mu_{1} \ldots \mu_{D-1}}$ and it transforms as scalar density under general coordinate transformations, which is evident from its definition (4). Accordingly, the integration element $\int d^{4} x \Phi(A)$ is manifestly invariant under general coordinate transformations.

Let us stress that the term "non-Riemannian" relates only to the nature of the volume element density (4), whose definition does not involve the metric. Otherwise the geometry of the spacetime is a regular Riemannian one - scalar products of vector fields are given as usual by the Riemannian metric $g_{\mu \nu}$, the connection $\Gamma_{\mu \nu}^{\lambda}$ is the usual Levi-Civita one in terms of $g_{\mu \nu}$, there is no torsion, etc.

\section{The action}

In general, modified gravity Lagrangian actions based on the non-Riemannian volume-form formalism have the follow- 
ing generic form (here and below we are using units with $\left.16 \pi G_{\text {Newton }}=1\right)$ :

$$
\begin{aligned}
S= & \int d^{4} x\left\{\Phi_{1}(A)\left[R+L^{(1)}\right]+\Phi_{2}(B)\right. \\
& {\left.\left[L^{(2)}+\frac{\Phi_{0}(C)}{\sqrt{-g}}\right]+\cdots\right\} . }
\end{aligned}
$$

Here $\Phi_{1}(A), \Phi_{2}(B), \Phi_{0}(C)$ are several different non-Riemannian volume element densities of the form (4), i.e., defined by auxiliary rank 3 tensor gauge fields $A_{\mu \nu \lambda}, B_{\mu \nu \lambda}$, $C_{\mu \nu \lambda} ; R$ denotes the scalar curvature in either first-order (Palatini) or second order (metric) formalism; $L^{(1)}$ and $L^{(2)}$ are some matter field Lagrangians; the dots indicate possible additional terms containing higher powers of the nonRiemannian volume element densities e.g., $\left(\Phi_{1}(A)\right)^{2} / \sqrt{-g}$. The specific forms of $L^{(1)}$ and $L^{(2)}$ can be uniquely fixed via the requirement for invariance of (5) under global Weyl-scale invariance (see (10) below).

Let us stress that the modified gravity action (5), in complete analogy with

$$
\int d^{4} x \sqrt{-g}[R+\ldots]
$$

which is the standard Einstein-Hilbert action, is explicitly invariant under general coordinate reparametrizations since, as mentioned above, non-Riemannian volume element densities transform as scalar densities similarly to $\sqrt{-g}$.

A characteristic feature of the modified gravitational theories (5) is that when starting in the first-order (Palatini) formalism all non-Riemannian volume-forms are almost puregauge degrees of freedom, i.e. they do not introduce any additional physical (field-propagating) gravitational degrees of freedom except for few discrete degrees of freedom with conserved canonical momenta appearing as arbitrary integration constants. The reason is that the modified gravity action in Palatini formalism is linear w.r.t. the velocities of some of the auxiliary gauge field components defining the nonRiemannian volume element densities, and does not depend on the velocities of the rest of auxiliary gauge field components. The (almost) pure-gauge nature of the latter is explicitly shown in Refs. [53,56] (appendices A) employing the standard canonical Hamiltonian treatment of systems with gauge symmetries, i.e., systems with first-class Hamiltonian constraints a'la Dirac (e.g., [60,61]).

Unlike Palatini formalism, the above situation changes significantly when we treat (5) in the second order (metric) formalism. In the latter case the "Einstein-Hilbert" part $\int d^{4} x \Phi_{1}(A) R$ of the modified gravity action (5) contains second order time derivative terms of the metric in $R$, which is in sharp contrast with the case of ordinary Riemannian volume element $\int d^{4} x \sqrt{-g} R$ where the corresponding secondorder time derivatives amount to a total derivative. According to the general canonical Hamiltonian treatment of systems with higher-order time derivatives on the canonical variables (see e.g., [62] - modern version of the classical Ostrogradsky formalism [63]) the presence of the latter implies the appearance of some of the corresponding velocities as additional physical degrees of freedom. In the present case this is reflected in the fact that (as we will see below, Eqs.(19)-(21)) upon passing to the physical Einstein frame via conformal transformation:

$g_{\mu \nu} \rightarrow \bar{g}_{\mu \nu}=\chi_{1} g_{\mu \nu} \quad, \quad \chi_{1} \equiv \frac{\Phi_{1}(A)}{\sqrt{-g}}$,

the first non-Riemannian volume element density $\Phi_{1}(A)$ in (5) is not any more a "pure gauge", but creates a new dynamical canonical scalar field $u$ via $\chi_{1}=\exp \frac{u}{\sqrt{3}}$. In the following Section we will see how a non-trivial inflationary potential for $u$ is dynamically generated.

\section{Einstein Frame: the Effective Scalar Potential}

Let us now consider the simplest member in the class of modified gravitational models (5) with no original matter fields. i.e., $L^{(1)}=0$ and $L^{(2)}=0$, and where we only add a quadratic term w.r.t. non-Riemannian volume element density $\Phi_{1}(A)$ :

$S=\int d^{4} x\left\{\Phi_{1}(A)\left[R-2 \Lambda_{0} \frac{\Phi_{1}(A)}{\sqrt{-g}}\right]+\Phi_{2}(B) \frac{\Phi_{0}(C)}{\sqrt{-g}}\right\}$,

Here $R$ is the scalar curvature in the second order (metric) formalism and:

$$
\begin{aligned}
& \Phi_{1}(A) \equiv \frac{1}{3 !} \varepsilon^{\mu \nu \kappa \lambda} \partial_{\mu} A_{\nu \kappa \lambda}, \Phi_{2}(B) \equiv \frac{1}{3 !} \varepsilon^{\mu \nu \kappa \lambda} \partial_{\mu} B_{\nu \kappa \lambda}, \\
& \Phi_{0}(C) \equiv \frac{1}{3 !} \varepsilon^{\mu \nu \kappa \lambda} \partial_{\mu} C_{\nu \kappa \lambda} .
\end{aligned}
$$

The specific form of the action (8) is dictated by the requirement about global Weyl-scale invariance under:

$$
\begin{aligned}
g_{\mu \nu} & \rightarrow \lambda g_{\mu \nu}, \\
A_{\mu \nu \kappa} & \rightarrow \lambda A_{\mu \nu \kappa}, B_{\mu \nu \kappa} \rightarrow \lambda^{2} B_{\mu \nu \kappa}, C_{\mu \nu \kappa} \rightarrow C_{\mu \nu \kappa} .
\end{aligned}
$$

where $\lambda=$ const.

Scale invariance has always played an important role since the original papers on the non-canonical volume-form formalism [49].

In a more general context let us recall, that scale invariance is a symmetry which relates small scales to large scales. As such (together with conformal symmetry) it plays fundamental role in quantum field theory and modern string theory in particle physics at (ultra)high energies as it dynamically generates (via spontaneous breakdown) mass scales hierarchies. On the other hand it plays an important role in cosmology as well, where it leads naturally to flat inflationary potentials 
(in the present context this is because it introduces a shift symmetry of the scalar field(s)) and produces candidates for dark matter (see the lectures at CERN's Workshop on "Scale invariance in particle physics and cosmology”, Ref. [64]). Also let us note another specific application of spontaneously broken dilatation symmetry in combination with application of the non-canonical volume form formalism: elimination of the Fifth Force Problem in a quintessential inflationary scenario [51].

The equations of motion resulting from (8) upon variation w.r.t. the auxiliary gauge fields $A_{\mu \nu \lambda}, B_{\mu \nu \lambda}, C_{\mu \nu \lambda}$ yield, respectively:

$$
\begin{aligned}
R-4 \Lambda_{0} \frac{\Phi_{1}(A)}{\sqrt{-g}} & =-M_{1} \equiv \text { const }, \\
\frac{\Phi_{0}(C)}{\sqrt{-g}} & =-M_{2} \equiv \text { const }, \quad \frac{\Phi_{2}(B)}{\sqrt{-g}}=\chi_{2} \equiv \text { const. }
\end{aligned}
$$

Here $M_{1}, M_{2}$ and $\chi_{2}$ are (dimensionful and dimensionless, respectively) integration constants. The appearance of $M_{1}, M_{2}$ indicate spontaneous breaking of global Weyl symmetry (10).

The equations of motion w.r.t. $g_{\mu \nu}$ from (8) read:

$$
\begin{gathered}
R_{\mu \nu}-\Lambda_{0} \chi_{1} g_{\mu \nu}+\frac{1}{\chi_{1}}\left(g_{\mu \nu} \square \chi_{1}-\nabla_{\mu} \nabla_{\nu} \chi_{1}\right) \\
-\frac{\chi_{2} M_{2}}{\chi_{1}} g_{\mu \nu}=0
\end{gathered}
$$

with $\chi_{1}$ as in (7). On the other hand, taking the trace of (13) and using Eq. (11) we obtain the equation of motion for $\chi_{1}$ :

$3 \frac{\square \chi_{1}}{\chi_{1}}-\frac{4 \chi_{2} M_{2}}{\chi_{1}}-M_{1}=0$.

We now transform Eqs. (13) and (14) via the conformal transformation (7) and show that the transformed equations acquire the standard form of Einstein equations w.r.t. the new "Einstein-frame" metric $\bar{g}_{\mu \nu}$. To this end we are using the known formulas for the conformal transformations of $R_{\mu \nu}$ and $\square \Psi$, the latter being an arbitrary scalar field, in particular $\Psi \equiv \chi_{1}$ (see e.g. Ref. [65]; bars indicate magnitudes in the $\bar{g}_{\mu \nu}$-frame):

$$
\begin{aligned}
R_{\mu \nu}(g)= & R_{\mu \nu}(\bar{g})-3 \frac{\bar{g}_{\mu \nu}}{\chi_{1}} \bar{g}^{\kappa \lambda} \partial_{\kappa} \chi_{1}^{1 / 2} \partial_{\lambda} \chi_{1}^{1 / 2} \\
& +\chi_{1}^{-1 / 2}\left(\bar{\nabla}_{\mu} \bar{\nabla}_{\nu} \chi_{1}^{1 / 2}+\bar{g}_{\mu \nu} \bar{\square} \chi_{1}^{1 / 2}\right),
\end{aligned}
$$

and

$$
\square \chi_{1}=\chi_{1}\left(\bar{\square} \chi_{1}-2 \bar{g}^{\mu \nu} \frac{\partial_{\mu} \chi_{1}^{1 / 2} \partial_{\nu} \chi_{1}}{\chi_{1}^{1 / 2}}\right),
$$

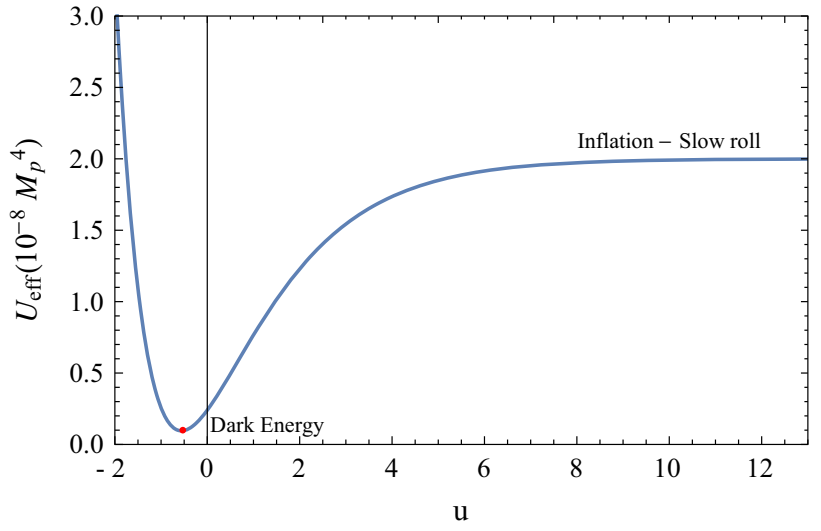

Fig. 1 Qualitative shape of the effective potential $U_{\text {eff }}(u)$ in the Einstein frame, as presented in Eq. (19). The physical unit for $u$ is $M_{\text {Planck }} / \sqrt{2}$

Following the analogous derivation in Ref. [66], upon using (15), (16) we rewite Eq. (13) as:

$$
\begin{aligned}
& R_{\mu \nu}(\bar{g})-\frac{1}{2} \bar{g}_{\mu \nu} R(\bar{g}) \\
& \quad=\frac{1}{2}\left[\partial_{\mu} u \partial_{\nu} u-\bar{g}_{\mu \nu}\left(\frac{1}{2} \bar{g}^{\kappa \lambda} \partial_{\kappa} u \partial_{\lambda} u+U_{\text {eff }}(u)\right)\right],
\end{aligned}
$$

where we have redefined:

$\Phi_{1}(A) / \sqrt{-g} \equiv \chi_{1}=\exp (u / \sqrt{3})$

in order to obtain a canonically normalized kinetic term for the scalar field $u$, and where:

$$
\begin{aligned}
U_{\text {eff }}(u)= & 2 \Lambda_{0}-M_{1} \exp \left(-\frac{u}{\sqrt{3}}\right) \\
& +\chi_{2} M_{2} \exp \left(-2 \frac{u}{\sqrt{3}}\right) .
\end{aligned}
$$

On the other hand, using (16) we rewrite Eq. (14) in terms of the canonical scalar field $u$ :

$\bar{\square} u+\frac{\partial U_{\text {eff }}}{\partial u}=0$

with $U_{\text {eff }}$ as in (19).

Accordingly, the corresponding Einstein-frame action reads:

$S_{\mathrm{EF}}=\int d^{4} x \sqrt{-\bar{g}}\left[R(\bar{g})-\frac{1}{2} \bar{g}^{\mu \nu} \partial_{\mu} u \partial_{\nu} u-U_{\mathrm{eff}}(u)\right]$.

We now observe an important result - in (21) we have a dynamically created scalar field $u$ with a non-trivial effective scalar potential $U_{\mathrm{eff}}(u)$ (19) entirely dynamically generated by the initial non-Riemannian volume elements in (8) because of the appearance of the free integration constants $M_{1}, M_{2}, \chi_{2}$ in their respective equations of motion (11), (12).

The qualitative shape of (19) is depicted on Fig. 1. The effective potential $U_{\text {eff }}(u)$ has two main features relevant 
for cosmological applications. First, $U_{\mathrm{eff}}(u)(19)$ possesses a flat region for large positive $u$ and, second, it has a stable minimum for a small finite value $u=u_{*}$ :

(i) $U_{\text {eff }}(u) \simeq 2 \Lambda_{0}$ for large $u$;

(ii) $\frac{\partial U_{\text {eff }}}{\partial u}=0$ for $u \equiv u_{*}$ where:

$$
\begin{aligned}
\exp \left(-\frac{u_{*}}{\sqrt{3}}\right) & =\frac{M_{1}}{2 \chi_{2} M_{2}},\left.\quad \frac{\partial^{2} U_{\mathrm{eff}}}{\partial u^{2}}\right|_{u=u_{*}} \\
& =\frac{M_{1}^{2}}{6 \chi_{2} M_{2}}>0 .
\end{aligned}
$$

The flat region of $U_{\text {eff }}(u)$ for large positive $u$ correspond to "early" universe' inflationary evolution with energy scale $2 \Lambda_{0}$. On the other hand, the region around the stable minimum at $u=u_{*}$ (22) correspond to "late" universe' evolution where the minimum value of the potential:

$U_{\mathrm{eff}}\left(u_{*}\right)=2 \Lambda_{0}-\frac{M_{1}^{2}}{4 \chi_{2} M_{2}} \equiv 2 \Lambda_{\mathrm{DE}}$

is the dark energy density value $[67,68]$.

Let us note that the effective potential $U_{\text {eff }}(19)$ generalizes the well-known Starobinsky inflationary potential [1] ((19) reduces to Starobinsky potential upon taking the following special values for the parameters: $\Lambda_{0}=\frac{1}{4} M_{1}=\frac{1}{2} \chi_{2} M_{2}$ ).

\section{Evolution of the homogeneous solution}

We now consider reduction of the Einstein-frame action (21) to the Friedmann-Lemaitre-Robertson-Walker (FLRW) setting with metric $d s^{2}=-N^{2} d t^{2}+a(t)^{2} d \mathbf{x}^{2}$, and with $u=u(t)$. In order to study the evolution of the scalar field $u=u(t)$ and the Friedmann scale factor $a=a(t)$, it is useful to use the method of autonomous dynamical systems.

The FLRW action describes a minimally coupled canonical scalar field $u$ with specific potential $U_{\text {eff }}(u)$ (19) (using again units with $16 \pi G_{\text {Newton }}=1$ ):

$$
\begin{aligned}
S_{\text {FLRW }}= & \int d^{4} x\left[-6 \frac{a \dot{a}^{2}}{N}+N a^{3}\left(\frac{1}{2} \dot{u}^{2} / N^{2}\right.\right. \\
& \left.\left.+M_{1} e^{-u / \sqrt{3}}-M_{2} \chi_{2} e^{-2 u / \sqrt{3}}\right)\right] .
\end{aligned}
$$

Variations w.r.t. $N, a, u$ (and subsequently using the gauge $N=1$ for the lapse function) yield the pertinent Friedmann and field equations ( $H=\dot{a} / a$ being the Hubble parameter):

$$
\begin{aligned}
& H^{2}=\frac{1}{6} \rho, \quad \rho=\frac{1}{2} \dot{u}^{2}+U_{\mathrm{eff}}(u), \\
& \dot{H}=-\frac{1}{4}(\rho+p), \quad p=\frac{1}{2} \dot{u}^{2}-U_{\mathrm{eff}}(u), \\
& \ddot{u}+3 H \dot{u}+\frac{\partial U_{\mathrm{eff}}}{\partial u}=0 .
\end{aligned}
$$

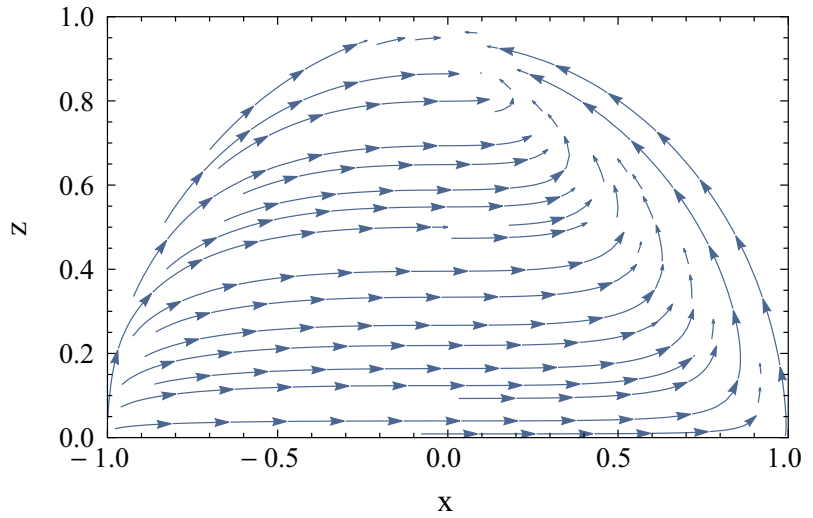

Fig. 2 Phase space portrait of the autonomous system (30). The $x$ axis denotes the relative kinetic part of the scalar inflaton, and the $z$ axis denotes the relative part of the dark energy density $\Lambda_{\mathrm{DE}}$

In the treatment of Eqs. (25)-(27) it is instructive to rewite them in terms of a set of dimensionless parameters (following the approach in Ref. [69]):

$x:=\frac{\dot{u}}{\sqrt{12} H}, \quad y:=\frac{\sqrt{U_{\mathrm{eff}}(u)-2 \Lambda_{\mathrm{DE}}}}{\sqrt{6} H}, \quad z:=\frac{\sqrt{\Lambda_{\mathrm{DE}}}}{\sqrt{3} H}$,

with $L_{\mathrm{DE}}$ as in (23). In these coordinates the system defines a closed orbit:

$x^{2}+y^{2}+z^{2}=1$,

which is equivalent to the first Friedmann equation (25).

Employing the variables $(x, y, z)$ in Eqs. (25)-(27) and taking into account the constraint (29) we obtain the autonomous dynamical system w.r.t. $(x, z)$ :

$$
\begin{aligned}
x^{\prime}= & \frac{\sqrt{3}}{2 \Lambda_{D E}} z^{2}\left[-M_{1} \xi(x, z)+2 M_{2} \chi_{2} \xi^{2}(x, z)\right] \\
& -3 x\left(1-x^{2}\right) \\
z^{\prime}= & 3 z x^{2}
\end{aligned}
$$

where the primes denote derivative w.r.t. the parameter $\mathcal{N}=$ $\log a$, and the function $\xi(x, z)$ is defined as:

$\xi(x, z)=\frac{M_{1}}{2 \chi_{2} M_{2}}\left[1-\sqrt{\frac{8 \Lambda_{0} M_{2} \chi_{2}}{M_{1}^{2}} \frac{1-x^{2}-z^{2}}{z^{2}}}\right]$.

The phase portrait of the system (30) is depicted on Fig. 2. There are two critical points in the system. The stable point $A(x=0, z=1)$ corresponds to the "late" universe de Sitter solution with the asymptotic cosmological constant $\Lambda_{\mathrm{DE}}$ (23).

The second point $B\left(x=0, z=\sqrt{\Lambda_{\mathrm{DE}} / \Lambda_{0}}\right)$ is unstable corresponding to the beginning of the universe' evolution in the "early" universe at large $u$. If the evolution starts at any point close to $B$, initially the evolution is of de Sitter type with effective cosmological constant $\approx \Lambda_{0}$. Then the dynamics 


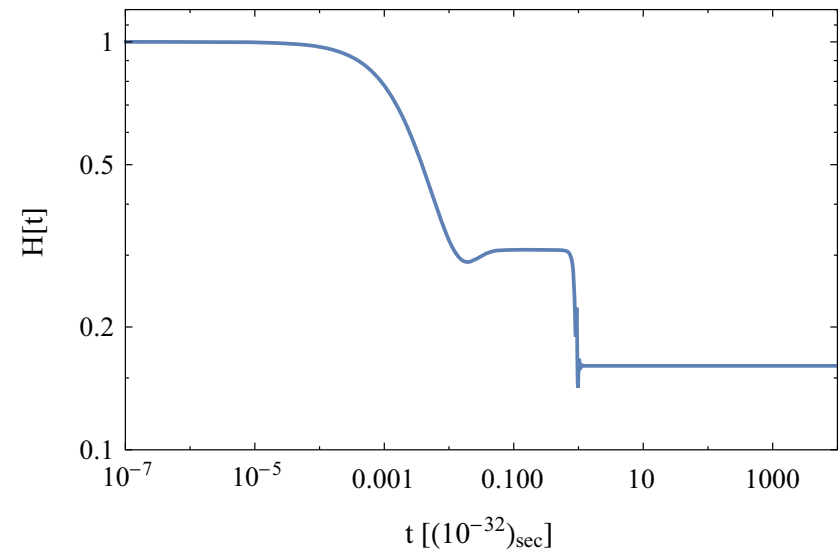

Fig. 3 Numerical example of the Hubble parameter $H(t)$ and the scalar field $u(t)$ vs. time. For short times the inflationary Hubble parameter is large and afterwards approaches its cosmological late time value. As an example we take: $\frac{M_{1}}{M_{2} \times_{2}} \simeq 10^{-2}, H(0) \sim 1, u(0) \sim 17$. The physical units for the numbers representing $u$ and $H$ on the vertical axis in both graphics conform to our choice of normalization of "Planck" units $16 \pi G_{\text {Newton }}=1$ in Eq. (5) and henceforth. Thus, the physical

drives the system away from $B$ all the way towards the stable point $A$ at late times.

Numerical solutions are demonstrated in Fig. 3. One can see that the Hubble parameter begins and ends with two different values. The first one is related to the inflationary epoch and the other related to the dark energy in the late universe. The scalar field $u$ oscillates around the minimum point $u_{*}$ (22) of $U_{\text {eff }}$ (19), which corresponds to particle creation in the reheating epoch.

\section{Perturbations}

In order to check the viability of the model we investigate the perturbations of the above background evolution, in particular focusing on the inflationary observables such as the scalar spectral index $n_{s}$ and the tensor-to-scalar ratio $r$. As usual, we introduce the Hubble slow-roll parameters [39,40], which in our case using the potential (19) read:

$$
\begin{gathered}
\epsilon=\left(\frac{U_{\mathrm{eff}}^{\prime}(u)}{U_{\mathrm{eff}}(u)}\right)^{2}=\frac{4 \zeta^{2}}{3} \frac{(1 / 2-\zeta)^{2}}{\left[(1 / 2-\zeta)^{2}+\delta / 4\right]^{2}}, \\
|\eta|=2\left|\frac{U_{\mathrm{eff}}^{\prime \prime}(u)}{U_{\mathrm{eff}}(u)}\right|=\frac{2 \zeta}{3} \frac{(1-4 \zeta)}{\left[(1 / 2-\zeta)^{2}+\delta / 4\right]},
\end{gathered}
$$

where:

$\zeta \equiv \frac{M_{2} \chi_{2}}{M_{1}} e^{-u / \sqrt{3}}, \quad \delta \equiv \frac{8 M_{2} \chi_{2}}{M_{1}^{2}} \Lambda_{\mathrm{DE}}$

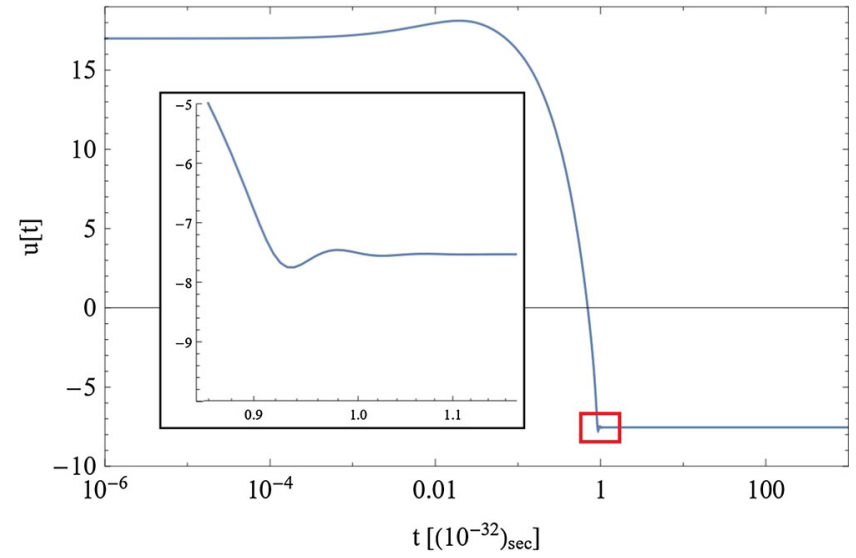

unit for $u$ is $1 / \sqrt{16 \pi G_{\text {Newton }}} \equiv M_{\text {Planck }} / \sqrt{2}$, and the unit for $H$ is around $10^{-17} \mathrm{sec}^{-1}$ or around $300(\mathrm{~km} / \mathrm{sec}) / \mathrm{Mpc}$ which conforms to the current value of the Hubble parameter around $60(\mathrm{~km} / \mathrm{sec}) / \mathrm{Mpc}$. On the right panel the blown-up rectangle depicts the oscillations of $u(t)$ around the minimum of $U_{\text {eff }}(19)$. One can see that the universe starts with an inflationary Hubble constant and ends with a smaller value representing the dark energy epoch

with $\Lambda_{\mathrm{DE}}$ - the dark energy density (23), and therefore $\delta$ very small.

Inflation ends when $\epsilon\left(u_{f}\right)=1$ for some $u=u_{f}$ where $\left(\zeta_{f} \equiv \frac{M_{2} \chi_{2}}{M_{1}} e^{-u_{f} / \sqrt{3}}\right)$ :

$$
\begin{aligned}
\zeta_{f} & =\frac{1}{2(1+2 / \sqrt{3})}\left[1+\frac{1}{\sqrt{3}}-\sqrt{1 / 3-(1+2 / \sqrt{3})} \delta\right] \\
& \simeq \frac{1}{2(1+2 / \sqrt{3})} .
\end{aligned}
$$

For the number of $e$-foldings $\mathcal{N}=\frac{1}{2} \int_{u_{i}}^{u_{f}} d u U_{\text {eff }} / U_{\text {eff }}^{\prime}$ we obtain:

$$
\begin{aligned}
\mathcal{N}= & \frac{3}{8}(1+\delta)\left(1 / \zeta_{i}-1 / \zeta_{f}\right) \\
& -\frac{3}{4}(1-\delta) \log \frac{\zeta_{f}}{\zeta_{i}}+\frac{3}{4} \delta \log \left(\frac{1-2 \zeta_{i}}{1-2 \zeta_{f}}\right),
\end{aligned}
$$

where $\zeta_{i} \equiv \frac{M_{2} \chi_{2}}{M_{1}} e^{-u_{i} / \sqrt{3}}$ and $u=u_{i}$ is very large corresponding to the start of the inflation. Ignoring $\delta$ and using the last equality (35) we have approximately:

$$
\begin{aligned}
\mathcal{N} \simeq & \frac{3 M_{1}}{8 M_{2} \chi_{2}} e^{u_{i} / \sqrt{3}}-\frac{\sqrt{3}}{4} u_{i}-\frac{3}{4}(1+2 / \sqrt{3}) \\
& +\frac{3}{4} \log (2(1+2 / \sqrt{3})) .
\end{aligned}
$$

Using the slow-roll parameters, one can calculate the values of the scalar spectral index and the tensor-to-scalar ratio respectively as $[45,70]$ :

$r \approx 16 \epsilon, \quad n_{s} \approx 1-6 \epsilon+2 \eta$ 


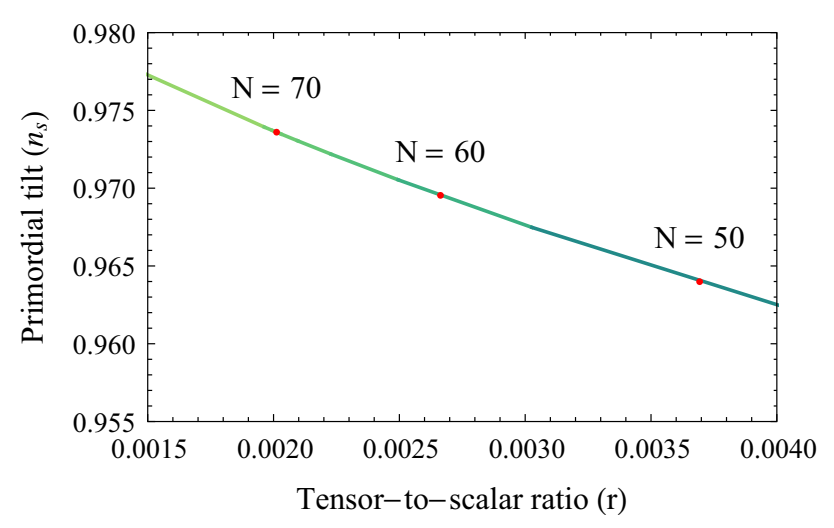

Fig. 4 The predicted values of the $r$ and $n_{s}$ for different $e$-foldings. The different values of the $r$ and $n_{s}$ are compatible with the observational data

Taking into account Eqs. (32),(33) (ignoring $\delta$ ) and (37) we find:

$$
\begin{aligned}
r & \simeq \frac{12}{\left[\mathcal{N}+\frac{\sqrt{3}}{4} u_{i}(\mathcal{N})+c_{0}\right]^{2}}, \\
c_{0} & \equiv \frac{\sqrt{3}}{2}-\frac{3}{4} \log (2(1+2 / \sqrt{3})) ;
\end{aligned}
$$

and

$n_{s} \simeq 1-\frac{r}{4}-\sqrt{\frac{r}{3}}$,

where $u_{i}(\mathcal{N})$ is the solution of the transcedental Eq. (37) for $u_{i}$ as a function of $\mathcal{N}$.

One viable example in our model is to take $\mathcal{N}=60 e$ folds. Equation (37) yields $\mathcal{N}=60$ provided we choose $\frac{M_{1}}{M_{2} \chi_{2}} \simeq 10^{-2}$, which yields $u_{i} \simeq 17$. In such a way the observables are predicted to be:

$n_{s} \approx 0.969, \quad r \approx 0.0026$,

which are well inside the PLANCK observed constraints [71]:

$0.95<n_{s}<0.97, \quad r<0.064$

Figure 4 demonstrates the relation between the number of $e$-folds and the dimensionless parameters. One can see that all those values fit the latest PLANCK collaboration constraints.

\section{Conclusions}

We propose a very simple gravity model without any initial matter fields in terms of several alternative non-Riemannian spacetime volume elements within the second order (metric) formalism. We show how the non-Riemannian volumeelements, when passing to the physical Einstein frame, create a canonical scalar field and produce dynamically a non-trivial inflationary-type potential for the latter with a large flat region and a stable low-lying minimum. We study the evolution of the cosmological solutions from the point of view of the theory of dynamical systems. Our model predicts scalar spectral index $n_{s} \approx 0.96$ and tensor-to-scalar ratio $r \approx 0.002$ for 60 $e$-folds, which is in accordance with the observational data.

A natural next step is to consider two-field inflation (Refs. [69,72-80], for a geometric treatment see Refs. [81-85], and references therein) by adding a new scalar field $\varphi$ with nontrivial potentials in the starting modified gravity action (8) built in terms of several non-Riemannian volume elements and subject to preserving the requirement of global Weylscale invariance (10).In this case the non-Riemannian volume elements will again generate a second scalar field $u$ and create dynamically a non-trivial two-field scalar potential with a very specific geometry of the field space of $\varphi, u$. This is studied in more detail in our subsequent work [78], where it is shown that the latter dynamically generated two-field inflationary model similarly conforms to the observational data.

Acknowledgements We gratefully acknowledge support of our collaboration through the Exchange Agreement between Ben-Gurion University, Beer-Sheva, Israel and Bulgarian Academy of Sciences, Sofia, Bulgaria. E.N. and S.P. are thankful for support by Contract DN 18/1 from Bulgarian National Science Fund. D.B., E.G. and E.N. are also partially supported by COST Actions CA15117, CA16104 and the action CA18108. D.B., E.N. and S.P. acknowledge illuminating discussions with Lilia Anguelova. Finally we would like to thank the referees whose comments contributed to significant improvement of the presentation.

Data Availability Statement This manuscript has no associated data or the data will not be deposited. [Authors' comment: All data generated or analyzed during this study are included in this published article.]

Open Access This article is distributed under the terms of the Creative Commons Attribution 4.0 International License (http://creativecomm ons.org/licenses/by/4.0/), which permits unrestricted use, distribution, and reproduction in any medium, provided you give appropriate credit to the original author(s) and the source, provide a link to the Creative Commons license, and indicate if changes were made. Funded by SCOAP . $^{3}$

\section{References}

1. A.A. Starobinsky, JETP Lett. 30, 682 (1979) [Pisma Zh. Eksp. Teor. Fiz. 30 (1979) 719]

2. A.A. Starobinsky, Phys. Lett. B 91, 99 (1980)

3. A.H. Guth, Phys. Rev. D 23, 347 (1981)

4. A.D. Linde, Phys. Lett. B 108, 389 (1982)

5. A. Albrecht, P.J. Steinhardt, Phys. Rev. Lett. 48, 1220 (1982)

6. V.F. Mukhanov, G.V. Chibisov, JETP Lett. 33, 532 (1981) [Pisma Zh. Eksp. Teor. Fiz. 33, 549 (1981)]

7. A.H. Guth, S.Y. Pi, Phys. Rev. Lett. 49, 1110 (1982)

8. S. Perlmutter et al., Supernova Cosmology Project Collaboration. Astrophys. J. 517, 565 (1999). arXiv:astro-ph/9812133

9. E.J. Copeland, M. Sami, S. Tsujikawa, Int. J. Mod. Phys. D 15, 1753 (2006). arXiv:hep-th/0603057 
10. S. Weinberg, Ultraviolet divergences in quantum theories of gravitation, in General Relativity. An Einstein Centenary Survey, ed. by S. Hawking, W. Israel (Cambridge University Press, Cambridge, 1979), pp. 790-831

11. M. Green, J. Schwarz, E. Witten, Superstring Theory, vol. 1 (Cambridge University Press, Cambridge, 1988)

12. S. Capozziello, V. Faraoni, Beyond Einstein Gravity: A Survey of Gravitational Theories for Cosmology and Astrophysics (Springer, Berlin, 2011)

13. S. Capozziello, M. De Laurentis, Phys. Rep. 509, 167 (2011). arXiv: 1108.6266

14. S. Nojiri, S. Odintsov, Phys. Rep. 505, 59 (2011)

15. E. Berti et al., Class. Quantum Gravity 32, 243001 (2016). arXiv:1501.07274

16. S. Nojiri, S. Odintsov, V. Oikonomou, Phys. Rep. 692, 1 (2017). arXiv: 1705.11098

17. G.R. Dvali, S.H.H. Tye, Phys. Lett. B 450, 72 (1999). arXiv:hep-ph/9812483

18. M. Kawasaki, M. Yamaguchi, T. Yanagida, Phys. Rev. Lett. 85, 3572 (2000). arXiv:hep-ph/0004243

19. M. Bojowald, Phys. Rev. Lett. 89, 261301 (2002). arXiv:gr-qc/0206054

20. S. Nojiri, S.D. Odintsov, Phys. Rev. D 68, 123512 (2003). arXiv:hep-th/0307288

21. S. Kachru, R. Kallosh, A.D. Linde, J.M. Maldacena, L.P. McAllister, S.P. Trivedi, JCAP 0310, 013 (2003). arXiv:hep-th/0308055

22. S. Nojiri, S.D. Odintsov, Gen. Relativ. Gravit. 38, 1285 (2006). arXiv:hep-th/0506212

23. R. Ferraro, F. Fiorini, Phys. Rev. D 75, 084031 (2007). arXiv:gr-qc/0610067

24. G. Cognola, E. Elizalde, S. Nojiri, S.D. Odintsov, L. Sebastiani, S. Zerbini, Phys. Rev. D 77, 046009 (2008). arXiv:0712.4017 [hep-th]

25. Y.F. Cai, E.N. Saridakis, Phys. Lett. B 697, 280 (2011). arXiv:1011.1245 [hep-th]

26. A. Ashtekar, D. Sloan, Gen. Relativ. Gravit. 43, 3619 (2011). arXiv:1103.2475 [gr-qc]

27. T. Qiu, E.N. Saridakis, Phys. Rev. D 85, 043504 (2012). arXiv:1107.1013 [hep-th]

28. F. Briscese, A. Marcian, L. Modesto, E.N. Saridakis, Phys. Rev. D 87(8), 083507 (2013). arXiv:1212.3611 [hep-th]

29. J. Ellis, D.V. Nanopoulos, K.A. Olive, Phys. Rev. Lett. 111, 111301 (2013) Erratum: [Phys. Rev. Lett. 111, no. 12, 129902 (2013)]. arXiv:1305.1247 [hep-th]

30. S. Basilakos, J.A.S. Lima, J. Sola, Int. J. Mod. Phys. D 22, 1342008 (2013). arXiv:1307.6251 [astro-ph.CO]

31. L. Sebastiani, G. Cognola, R. Myrzakulov, S.D. Odintsov, S. Zerbini, Phys. Rev. D 89(2), 023518 (2014). arXiv:1311.0744 [grqc]

32. D. Baumann, L. McAllister, arXiv:1404.2601 [hep-th]

33. I. Dalianis, F. Farakos, JCAP 1507(07), 044 (2015). arXiv: 1502.01246 [gr-qc]

34. P. Kanti, R. Gannouji, N. Dadhich, Phys. Rev. D 92(4), 041302 (2015). arXiv:1503.01579 [hep-th]

35. M. De Laurentis, M. Paolella, S. Capozziello, Phys. Rev. D 91(8), 083531 (2015). arXiv:1503.04659 [gr-qc]

36. S. Basilakos, N.E. Mavromatos, J. Sola Universe 2(3), 14 (2016). arXiv: 1505.04434 [gr-qc]

37. A. Bonanno, A. Platania, Phys. Lett. B 750, 638 (2015). arXiv: 1507.03375 [gr-qc]

38. A.S. Koshelev, L. Modesto, L. Rachwal, A.A. Starobinsky, JHEP 1611, 067 (2016). arXiv:1604.03127 [hep-th]

39. J. Martin, C. Ringeval, V. Vennin, Phys. Dark Univ. 5-6, 75 (2014). arXiv:1303.3787 [astro-ph.CO]

40. K. Bamba, S.D. Odintsov, E.N. Saridakis, Mod. Phys. Lett. A 32(21), 1750114 (2017). arXiv:1605.02461 [gr-qc]
41. H. Motohashi, A.A. Starobinsky, Eur. Phys. J. C 77(8), 538 (2017). arXiv: 1704.08188 [astro-ph.CO]

42. V.K. Oikonomou, Int. J. Mod. Phys. D 27(05), 1850059 (2018). arXiv: 1711.03389 [gr-qc]

43. I. Antoniadis, A. Karam, A. Lykkas, K. Tamvakis, JCAP 1811(11), 028 (2018). arXiv:1810.10418 [gr-qc]

44. A. Karam, T. Pappas, K. Tamvakis, arXiv:1903.03548 [gr-qc]

45. S. Nojiri, S.D. Odintsov, E.N. Saridakis, arXiv:1904.01345 [gr-qc]

46. D. Benisty, E.I. Guendelman, E.N. Saridakis, H. Stoecker, J. Struckmeier, D. Vasak, Phys. Rev. D 100(4), 043523 (2019). arXiv: 1905.03731 [gr-qc]

47. E.I. Guendelman, A. Kaganovich, Phys. Rev. D 53, 7020 (1996). arXiv:gr-qc/9605026

48. F. Gronwald, U. Muench, A. Macias, F. Hehl, Phys. Rev. D 58, 084021 (1998). arXiv:gr-qc/9712063

49. E.I. Guendelman, Mod. Phys. Lett. A 14, 1043-1052 (1999). arXiv:gr-qc/9901017

50. E.I. Guendelman, A. Kaganovich, Phys. Rev. D 60, 065004 (1999). arXiv:gr-qc/9905029

51. E.I. Guendelman, A.B. Kaganovich, Ann. Phys. 323, 866 (2008). arXiv:0704.1998

52. E.I. Guendelman, E. Nissimov, S. Pacheva, Bulg. J. Phys. 41, 123 (2014). arXiv:1404.4733

53. E.I. Guendelman, E. Nissimov, S. Pacheva, Int. J. Mod. Phys. A 30, 1550133 (2015). arXiv:1504.01031

54. E.I. Guendelman, E. Nissimov, S. Pacheva, Eur. J. Phys. C 75, 472-479 (2015). arXiv: 1508.02008

55. E.I. Guendelman, E. Nissimov, S. Pacheva, Eur. J. Phys. C 76, 90 (2016). arXiv: 1511.07071

56. E.I. Guendelman, E. Nissimov, S. Pacheva, Int. J. Mod. Phys. D 25, 1644008 (2016). arXiv:1603.06231

57. E.I. Guendelman, E. Nissimov, S. Pacheva, Springer Proceedings in Mathematics and Statistics v.225, in Quantum Theory and Symmetries with Lie Theory and Its Applications in Physics, vol. 2, ed. by V. Dobrev (Springer, Berlin, 2018). arXiv:1712.09844

58. E.I. Guendelman, E. Nissimov, S. Pacheva, AIP Conference Proceedings 2075, 090030 (2019). arXiv: 1808.03640

59. M. Spivak, Calculus on Manifolds: A Modern Approach to Classical Theorems of Advanced Calculus, Ch. 5 (CRC Press, Boca Raton, 2018), p. 126

60. M. Henneaux, C. Teitelboim, Quantization of Gauge Systems (Princeton University Press, Princeton, 1991)

61. H. Rothe, K. Rothe, Classical and Quantum Dynamics of Constrained Hamiltonian Systems, Ch. 3 (World Scientific Publishing, New York, 2010)

62. D. Gitman, I. Tyutin, Quantization of Fields with Constraints, Ch. 7.1 (Springer, Berlin, 1990)

63. M. Ostrogradski, Mem. Ac. St. Petersbourg VI4 (1850) 385

64. CERN Workshop on "Scale invariance in particle physics and cosmology" (Jan 28-Feb 01, 2019), https://indico.cern.ch/event/ 740038/timetable/\#all

65. M. Dabrowski, J. Garecki, D. Blaschke, Ann. Phys. 18, 13 (2009). arXiv:0806.2683

66. D. Benisty, E.I. Guendelman, Class. Quantum Gravity 36, 095001 (2019). arXiv: 1809.09866

67. C. R. Angus et al. [DES Collaboration], Mon. Not. R. Astron. Soc. 487(2), 2215 (2019). arXiv:1812.04071 [astro-ph.HE]

68. Y. Zhang et al. [DES Collaboration], Astrophys. J. 874(2), 165 (2019). arXiv:1812.04004 [astro-ph.CO]

69. S. Bahamonde, C.G. Bhmer, S. Carloni, E.J. Copeland, W. Fang, N. Tamanini, Phys. Rep. 775-777, 1 (2018). arXiv:1712.03107 [gr-qc]

70. I. Dalianis, A. Kehagias, G. Tringas, JCAP 1901, 037 (2019). arXiv: 1805.09483 [astro-ph.CO]

71. Y. Akrami et al. [Planck Collaboration], arXiv:1807.06211 [astroph.CO] 
72. A. Linde, Phys. Lett. B 259, 38 (1991)

73. A. Linde, Phys. Rev. D 49, 748 (1994)

74. E. Copeland, A. Liddle, D. Lyth, E. Stewart, D. Wands, Phys. Rev. D 49, 6410 (1994)

75. I. Ben-Dayan, Phys. Rev. D 99(10), 101301 (2019). arXiv:1808.01615 [hep-th]

76. M. Artymowski, I. Ben-Dayan, JCAP 1905(05), 042 (2019). arXiv:1902.02849 [gr-qc]

77. I. Ben-Dayan, S. Jing, M. Torabian, A. Westphal, L. Zarate, JCAP 1409, 005 (2014). arXiv:1404.7349 [hep-th]

78. D. Benisty, E.I. Guendelman, E. Nissimov, S. Pacheva, arXiv:1907.07625 [astro-ph.CO]
79. V. Aragam, S. Paban, R. Rosati, arXiv:1905.07495 [hep-th]

80. J. Kubo, M. Lindner, K. Schmitz, M. Yamada, Phys. Rev. D 100(1), 015037 (2019). arXiv:1811.05950 [hep-ph]

81. L. Anguelova, E.M. Babalic, C. Lazaroiu, arXiv:1905.01611

82. J.E. Madriz Aguilar, J. Zamarripa, M. Montes , C. Romero, arXiv: 1908.06220 [gr-qc]

83. A.O. Barvinsky, N. Kolganov, arXiv:1908.05697 [gr-qc]

84. S. Ahmad, A. De Felice, N. Jaman, S. Kuroyanagi, M. Sami, arXiv: 1908.03742 [gr-qc]

85. L.N. Granda, D.F. Jimenez, arXiv:1907.06806 [hep-th] 